\title{
CHARACTERIZATIONS OF FAVARD CLASSES FOR FUNCTIONS OF SEVERAL VARIABLES
}

BY P. L. BUTZER AND E. GÖRLICH

Communicated by Richard Arens, August 21, 1967

1. Introduction. There are two general approaches to the study of saturation theory (for the definitions see [5]), namely the integral transform method (cf. [3], [5]) and the semigroup method (cf. [4]). In this note a third method, a distribution theoretical method will be employed, in particular to characterize the Favard (saturation) classes defined by

$$
V_{\alpha}^{p}=\left\{f ; f(x) \in L^{p}\left(E^{n}\right),|v|^{\alpha} f^{\wedge}(v)=g^{\wedge}(v), g \in L^{p}\left(E^{n}\right)\right\} .
$$

Here $x, v$ denote vectors in $E^{n}$ with $|v|=\left(v_{1}^{2}+\cdots+v_{n}^{2}\right)^{1 / 2}$ and $\alpha$ a positive parameter. $f^{\wedge}(v)$ being the Fourier transform of $f$, this definition of $V_{\alpha}^{p}$ is meaningful only for $1<p \leqq 2$. In order to extend it to $2<p<\infty$ we use the classes of Bessel potentials

(2) $L_{\alpha}^{p}=\left\{f ; f \in L^{p}\left(E^{n}\right),\left(1+|v|^{2}\right)^{\alpha / 2} f^{\wedge}(v)=h^{\wedge}(v), h \in L^{p}\left(E^{n}\right)\right\}$.

This definition bears sense not only for $1<p \leqq 2$ but also for $2<p<\infty$ if the Fourier transform is taken in the distribution theoretical sense since $\left(1+|v|^{2}\right)^{\alpha / 2}$ is an infinitely differentiable and slowly increasing function (in the terminology of L. Schwartz [10]). The problem is to show the equivalence of (1) and (2) for $1<p \leqq 2$ and to give simple characterizations of (2), e.g. in terms of differentiability properties both in the classical and the distributional (or Sobolev) sense. An equivalent definition of the classes $L_{\alpha}^{p}$, investigated in [1], [2], [6], is given by

$$
L_{\alpha}^{p}=\left\{f ; f \in L^{p}\left(E^{n}\right), f=G_{\alpha} * h, h \in L^{p}\left(E^{n}\right)\right\},
$$

where $G_{\alpha}(x)$ is defined through $\hat{G_{\alpha}}(v)=\left(1+|v|^{2}\right)^{-\alpha / 2}$, having the properties $G_{\alpha}(x) \in L^{1}\left(E^{n}\right), G_{\alpha}(x) \geqq 0, \int_{E^{n}} G_{\alpha}(x) d x=(2 \pi)^{n / 2} . L_{\alpha}^{p}$ is a subspace of the space of tempered distributions.

For integral values of $\alpha$ we obtain an equivalence between $L_{\alpha}^{p}$ and the Sobolev space

$$
\begin{array}{r}
W_{\alpha}^{p}=\left\{f ; f \in L^{p}\left(E^{n}\right), D^{k} f \in L^{p}\left(E^{n}\right) \quad \text { for every } k=\left(k_{1}, \cdots, k_{n}\right)\right. \\
\text { with } \left.k_{j} \geqq 0 \text { and }|k|=\sum_{j=1}^{n} k_{j} \leqq \alpha\right\} .
\end{array}
$$


Here $D^{k} f=\partial^{|k|} f / \partial x_{1}^{k 1} \cdots \partial x_{n}^{k_{n}}$ means the distribution derivative of $f$.

2. Characterizations for $1<p<\infty, \alpha>0$.

THEOREM 1. For $1<p \leqq 2$ and $\alpha>0, f \in V_{\alpha}^{p}$ if and only if $f \in L_{\alpha}^{p}$.

The proof depends upon a lemma in a paper of E. M. Stein [11] where also some further characterizations of the class $L_{\alpha}^{p}$ are given for $0<\alpha<2$. Theorem 1 states that $L_{\alpha}^{p}$ is a continuation of $V_{\alpha}^{p}$ for $p>2$. Thus it is sufficient to give equivalent characterizations of $L_{\alpha}^{p}$. As a second step, using known results of A. P. Calderón [6], N. Aronszajn, F. Mulla and P. Szeptycki [2] we have

Theorem 2. Let $\alpha=1,2, \cdots$ and $1<p<\infty$. Then $f \in L_{\alpha}^{p}$ if and only if $f \in W_{\alpha}^{p}$.

Theorems 1, 2 enable us to prove many other characterizations of $V_{\alpha}^{p}$ and $L_{\alpha}^{p}$ for special values of $\alpha$ or $p$, especially those given in terms of ordinary derivatives. The cases $\alpha=1,2$ are the most important examples in the applications to saturation theory.

3. The case $\alpha=2$. The following list of equivalences is a consequence of Theorems 1,2 and of results of R. J. Nessel [8].

Theorem 3. Let $f \in L^{p}\left(E^{n}\right), 1<p<\infty$. The following assertions are equivalent:

(a) $f \in V_{2}^{p}$ (here the definition of $V_{2}^{p}$ is extended for $p>2$ by $|v|{ }^{2} f^{\wedge}(v)$ $=g^{\wedge}(v), g \in L^{p}\left(E^{n}\right)$, in the distributional sense);

(b) $f \in L_{2}^{p}$;

(c) $f \in W_{2}^{p}$;

(d) $\Delta f=g, g \in L^{p}\left(E^{n}\right) \quad\left(\Delta f=\partial^{2} f / \partial x_{1}^{2}+\cdots+\partial^{2} f / \partial x_{n}^{2}\right.$ in the distributional sense);

(e) for $j, k=1,2, \cdots, n$ the functions $f, \partial f / \partial x_{j}$ are absolutely continuous in each variable, and $\partial f / \partial x_{j}, \partial^{2} f / \partial x_{j} \partial x_{k} \in L^{p}\left(E^{n}\right)$ (the derivatives to be understood in the ordinary sense);

(f) for $j=1,2, \cdots, n$ (with $e_{j}=$ unit vector in $j$-direction)

$$
\left\|f\left(x+2 h e_{j}\right)-2 f\left(x+h e_{j}\right)+f(x)\right\|_{p}=O\left(h^{2}\right) \quad\left(h \in E^{1}, h \rightarrow 0\right) ;
$$

(g) $\|f(x+2 u)-2 f(x+u)+f(x)\|_{p}=O\left(|u|^{2}\right) \quad\left(u \in E^{n},|u| \rightarrow 0\right)$;

(h) $\sum \sum f(x+j h)-2^{n} f(x) \|_{p}=O\left(h^{2}\right) \quad(h \rightarrow 0)$,

where the sum runs over all $j=\left(j_{1}, \cdots, j_{n}\right)$ with $j_{k}= \pm 1$.

4. The case $\alpha=1$. The Hilbert transform of a function $f \in L^{p}\left(E^{n}\right)$, $1<p<\infty$, with respect to $x_{j}$ is defined by 


$$
\begin{array}{r}
\tilde{f_{j}}(x)=\lim _{\epsilon \rightarrow 0+} \frac{\Gamma((n+1) / 2)}{\pi^{(n+1) / 2}} \int_{|x-u| \geq \epsilon} f(u) \frac{x_{j}-u_{j}}{|x-u|^{n+1}} d u \\
(j=1,2, \cdots, n) .
\end{array}
$$

The functions $f_{f}^{\sim}(x)$ form the coordinates of a vector $(H f)(x)$ $=\sum_{j=1}^{n} e_{j} f_{j}^{\sim}(x)$ (cf. [7]) and again belong to $L^{p}\left(E^{n}\right)$. We have, in the case $\alpha=1$,

Theorem 4. Let $f \in L^{p}\left(E^{n}\right), 1<p<\infty$. The following statements are equivalent:

(a) $f \in L_{1}^{p}$,

(b) $f_{j} \in L_{1}^{p}$ for $1 \leqq j \leqq n$,

(c) $f \in W_{1}^{p}$,

(d) $(\operatorname{div} H f)(x)=\sum_{j=1}^{n}\left(\partial / \partial x_{j}\right) f_{j}^{\sim}(x) \in L^{p}\left(E^{n}\right)$ (distributional derivatives),

(e) for $j=1,2, \cdots, n$ the functions $f_{j}^{\sim}(x)$ are absolutely continuous in each variable, and the ordinary derivatives $\partial f_{j}^{\sim} / \partial x_{k}(k=1, \cdots, n)$ are in $L^{p}\left(E^{n}\right)$,

(f) for $j, k=1,2, \cdots, n$,

$$
\left\|\tilde{f_{j}}\left(x+h e_{k}\right)-\tilde{f_{j}}(x)\right\|=O(|h|) \quad(h \rightarrow 0) ;
$$

(g) $\left.\left\|f\left(x+h e_{j}\right)-f(x)\right\|=O(|h|) h \rightarrow 0 ; j=1,2, \cdots, n\right)$.

5. The case $p=2$. For $p=2$ many known equivalences between $L_{\alpha}^{2}$ and "fractional" Sobolev spaces or various types of Besov spaces can be applied to give new characterizations of $V_{\alpha}^{2}$ for arbitrary $\alpha>0$. For instance the fractional Sobolev spaces defined for fractional $\alpha=m+\beta(m=0,1,2, \cdots ; 0<\beta<1)$ by

$$
\begin{aligned}
W_{\alpha}^{2}=\left\{f ; f \in W_{m}^{2},\left[\int_{E^{n}} \frac{\left\|D^{k} f(\cdot+u)-D^{k} f(\cdot)\right\|_{2}^{2}}{|u|^{2 \beta+n}} d u\right]^{1 / 2}<\infty\right. \\
\left.\quad \text { for every } k=\left(k_{1}, \cdots, k_{n}\right) \text { with }|k|=m\right\}
\end{aligned}
$$

and for $\alpha=1,2, \cdots$ by (4) (see [9], [2, p. 74]) are equivalent to $V_{\alpha}^{2}$ for $\alpha>0$.

The present results are also connected with the theory of intermediate spaces which is presented in [4]. A detailed discussion of these results, including proofs and various extensions, will be published elsewhere. 


\section{REFERENCES}

1. N. Aronszajn and K. T. Smith, Theory of Bessel potentials. Part I, Ann. Inst. Fourier (Grenoble) 11 (1961), 385-475.

2. N. Aronszajn, F. Mulla and P. Szeptycki, On spaces of potentials connected with $L^{p}$ classes, Ann. Inst. Fourier (Grenoble) 13 (1963), 211-306.

3. P. L. Butzer, Fourier transform methods in the theory of approximation, Arch. Rational Mech. Anal. 5 (1960), 390-415.

4. P. L. Butzer and H. Berens, Semi-groups of operators and approximation, Grundlehren d. Math. Wiss., vol. 145, Springer, New York, 1967.

5. P. L. Butzer and R. J. Nessel, Favard classes for n-dimensional singular integrals, Bull. Amer. Math. Soc. 72 (1966), 493-498.

6. A. P. Calderon, Lebesgue spaces of differentiable functions and distributions, Proc. Sympos. Pure Math., Vol. 4, Amer. Math. Soc., Providence, R. I., 1961, 33-49.

7. J. Horvath, Transformadas de Hilbert de distribuciones, Segundo symposium sobre algunos problemas matematicos que se están estudiando en Latino America, Villavicencio-Mendoza, 1954, 61-70.

8. R. J. Nessel, Das Saturationsproblem für mehrdimensionale singuläre Integrale und seine Lösung mit Hilfe der Fouriertransformation, Doctoral dissertation, Aachen, 1965,145 pp.

9. S. M. NikolskiY, On imbedding, continuation and approximation theorems for differentiable functions of several variables, Russian Math. Surveys (5) 16 (1961), 55104.

10. L. Schwartz, Thêorie des distributions. I, II, Hermann, Paris, 1957.

11. E. M. Stein, The characterization of functions arising as potentials, Bull. Amer. Math. Soc. 67 (1961), 102-104.

The Technological University of Aachen, Germany 